Bundesgesundheitsbl 2018 $61: 446$

https://doi.org/10.1007/s00103-018-2715-1

Online publiziert: 21. März 2018

(c) Springer-Verlag GmbH Deutschland, ein Teil von Springer Nature 2018

CrossMark

Kommission für Krankenhaushygiene und Infektionsprävention (KRINKO) beim Robert Koch-Institut

Berlin, Deutschland

\title{
Erratum zu: Prävention von Infektionen, die von Gefäßkathetern ausgehen
}

\section{Teil 1 - Nichtgetunnelte zentralvenöse Katheter \\ Empfehlung der Kommission für Krankenhaushygiene und Infektionsprävention (KRINKO) beim Robert Koch-Institut}

(z. B. Isopropanol) mit CHX $2 \%$

(Kat. IB) oder Octenidin $0,1 \%$

(Kat. II) empfohlen.

https://doi.org/10.1007/s00103-016-

2487-4

Im Kapitel 3.2. Maßnahmen bei Anlage eines ZVK (maximale Barrieremaßnahmen und Hautantiseptik) heißt es:

„Die Kommission empfiehlt:

- Für die Hautantiseptik vor Anlage eines ZVK wird die Kombination eines alkoholischen Antiseptikums (z. B. Isopropanol) mit CHX 2\% oder Octenidin 0,1\% empfohlen (Kat. IA).“

Die Evidenzkategorie (IA) ist nicht korrekt. Wir danken den aufmerksamen Leserinnen und Lesern für diesen wichtigen Hinweis.

Die KRINKO hat nach einer erneuten Literaturrecherche [1-7] am 15.12.2017 hierüber beraten und passt die Evidenzkategorie wie folgt an:

- Für die Hautantiseptik vor Anlage eines ZVK wird die Kombination eines alkoholischen Antiseptikums

Die Online-Version des Originalartikels ist unter https://doi.org/10.1007/s00103-016-2487-4zu finden.
Die KRINKO weist außerdem darauf hin, dass sie trotz der unterschiedlichen Evidenz aus klinischen Studien auf Grund der vorliegenden Wirksamkeitsnachweise (Gutachten) beide Kombinationspräparate für gleichermaßen geeignet hält.

\section{Korrespondenzadresse}

Kommission für Krankenhaushygiene und Infektionsprävention (KRINKO) beim

Berlin, Deutschland

\section{Literatur}

1. Maki DG (2014) Chlorhexidine's role in skin antisepsis: questioning the evidence-Author's reply. Lancet 384(9951):1345-1346

2. Maki DG, Ringer M, Alvarado CJ (1991) Prospective randomised trial of povidone-iodine, alcohol, and chlorhexidine for prevention of infection associated with central venous and arterial catheters. Lancet 338(8763):339-343

3. Lai NM, Lai NA, O'Riordan E, Chaiyakunapruk N, Taylor JE, Tan K (2016) Skin antisepsis for reducing central venous catheter-related infections. Cochrane Database Syst Rev. https:// doi.org/10.1002/14651858.CD010140.pub2 Robert Koch-Institut
4. Mimoz O, Lucet JC, Kerforne T et al (2015) Skin antisepsis with chlorhexidine-alcohol versus povidone iodine-alcohol, with and without skin scrubbing, for prevention of intravascularcatheter-related infection (CLEAN): an open-label, multicentre, randomised, controlled, two-by-two factorial trial. Lancet 386(10008):2069-2077

5. Pages J, Hazera P, Megarbane B et al (2016) Comparison of alcoholic chlorhexidine and povidoneiodine cutaneous antiseptics for the prevention of central venous catheter-related infection: a cohort and quasi-experimental multicenter study. Intensive Care Med 42(9):1418-1426

6. Chaiyakunapruk N, Veenstra DL, Lipsky BA, Saint $S$ (2002) Chlorhexidine compared with povidoneiodine solution for vascular catheter-site care: a meta-analysis. Ann Intern Med 136(11):792-801

7. Dettenkofer M, Wilson C, Gratwohl A et al (2010) Skin disinfection with octenidine dihydrochloride for central venous catheter site care: a doubleblind, randomized, controlled trial. Clin Microbiol Infect 16(6):600-606 